Pierre-Alain Duc, Jonathan Braine and Elias Brinks, eds.

\title{
Stellar Populations of a Sample of Tidal Dwarf Galaxies
}

\author{
Peter M. Weilbacher \\ Department of Physics, Durham University, South Road, Durham, \\ DH1 3LE, UK \\ Uta Fritze-v. Alvensleben \\ Universitäts-Sternwarte Göttingen, Geismarlandstr. 11, D-37083 \\ Göttingen, Germany \\ Pierre-Alain Duc \\ CNRS FRE 2591 and CEA, DSM, DAPNIA, Service d'Astrophysique, \\ Centre d'Etudes de Saclay, 91191 Gif-sur-Yvette Cedex, France
}

\begin{abstract}
We investigate the stellar populations of a sample of Tidal Dwarf Galaxies, combining observations and evolutionary synthesis models to try and reveal their formation mechanism. On optical images we select a first sample of TDGs for which optical spectroscopy is used to measure metallicities and velocity structure. Finally, we estimate ages, burst strengths, and stellar masses from near-infrared imaging in comparison with a dedicated grid of evolutionary synthesis models, to assess if Tidal Dwarfs are formed out of collapsing gas clouds or by an accumulation of old stars from the parent galaxy or by a combination of both.
\end{abstract}

\section{Introduction \& Motivation}

The old idea of Zwicky(1956) that (dwarf) galaxies could be formed during encounters of giant galaxies has received a lot of observational support within the last decade (Duc \& Mirabel, 1994, Duc \& Mirabel, 1998, Hibbard et al., 1994). This data revealed the so called Tidal Dwarf Galaxies (TDGs) to be gas rich, dwarf galaxy sized knots with stellar masses of $10^{6} \ldots 10^{8} \mathrm{M}_{\odot}$ made of recycled material, still embedded in the tidal tail of their parent galaxy. Often, a young starburst on top of an older stellar population is observed. This is widely interpreted as formation of a new galaxy "in situ" in the tidal tails.

To tell the phenomenon of TDGs apart from e.g. super star clusters or chance alignments of matter, the following definition of TDGs was introduced (Duc et al., 2000; Weilbacher \& Duc, 2001):

A Tidal Dwarf Galaxy is a self-gravitating object with the mass of a dwarf galaxy, formed from recycled material that was expelled from a big galaxy in the course of tidal interaction.

Why are TDGs thought to be significant? First, they represent a special mode of galaxy formation which can be observed in the local universe, i.e. much more detailed than high-redshift galaxies forming in the early universe. Second, 
part of the dwarf galaxy populations of the Local Group or nearby galaxy clusters may have been formed as TDGs (Okazaki \& Taniguchi, 2000). And finally, they may explain part of the faint blue galaxy population at intermediate redshifts. At those times, the merger rate was higher than today (Conselice et al., 2003) and the galaxies were more gas rich, so that a large number of bright TDGs could have been formed.

But how do TDGs form? Are they preferentially built as stellar knots of which there may be several per merger as seen in the dynamical models of Barnes \& Hernquist (1992), or from giant gas clouds at the tips of the tidal tails which then afterwards may also attract stars into their potential as in the simulations of Elmegreen et al. (1993)? This question was the original starting point of our project. We therefore investigate the stellar populations of a sample of TDGs in order to find out which of these models is realized or more common in nature.

\section{Sample \& Approach}

Our sample of interacting galaxies consists of 14 interacting/merging galaxies from the catalog of Arp \& Madore (1987). It includes interacting galaxies of all Hubble types from ellipticals to irregular galaxies, some of which already in an advanced stage of merging. They have distances of $\sim 50$ to $370 \mathrm{Mpc}^{1}$ and were selected on the basis of their disturbed appearance. They do not, however, show especially long tidal tails or prominent knots on images of the Digital Sky Survey, as some of the other galaxies which had TDGs investigated previously.

Our analysis consists of three observational stages, each step is analyzed by means of evolutionary synthesis models: optical imaging for sample selection of TDG candidates, optical spectroscopy of these candidate TDGs to check their physical association with the interacting system and to investigate the properties and kinematics of the ionized gas, and, finally, near-infrared imaging to determine burst strengths, ages, and stellar masses of the TDGs. The observations were carried out with the ESO NTT, the ESO-3.6m telescope, and the FORS2 instrument on the VLT.

We use a version of the Göttingen Galaxy Evolution code GALEV for our modeling (for the details of the models see Weilbacher et al., 2000; Weilbacher, 2002). Here, we just recall that we model a starburst of varying strength on top of an old stellar population, and compute the optical to near-infrared broad-band luminosities and spectra of these composite populations at different ages before and after the burst maximum. We note that our code also includes the effects of gaseous emission lines and continuum on the spectra and broad band colors, which is an important ingredient as it can contribute up to $70 \%$ of the luminosity in optical broad band filters in the strong starbursts that we investigate here. The burst strength, which we define as the mass ratio

$$
b=\frac{M(\text { young stars })}{M(\text { all stars })}
$$

\footnotetext{
${ }^{1}$ converted from their heliocentric velocities using $H_{0}=75 \mathrm{~km} \mathrm{~s}^{-1} \mathrm{Mpc}^{-1}$
} 
is a central property in our investigation. Our grid of models covers burst strengths in the range from $8 \%$ to $100 \%$ of young stars.

\section{Results}

In the following we describe in more detail the procedure and results of our three stage approach to select and characterize Tidal Dwarfs.

\subsection{Optical Imaging \& Models}

Our optical $B, V, R$ imaging data was analyzed with aperture photometry yielding color and (absolute) magnitude information on more than 100 knots in the tidal tails of our sample of interacting galaxies. To select the best candidates for TDGs we compared this data with our models in two-color diagrams $(B-V$ vs. $V-R)$. Many objects turned out to be background objects on the basis to their colors, several knots were excluded as TDG candidates because of their location too close to the parent nuclei. We selected 44 good candidates for TDGs with luminosities $-17.0<M_{B}<-10.5$ mag.

At this stage we had to make assumptions about metallicity and extinction in the TDG candidates, so we could only derive first order estimates of burst ages and strengths. Observed color could only be matched by models with fairly high burst strengths, $b$ up to $20 \%$. We therefore expect strong fading after the TDGs exhaust their gas supplies and terminate their current starbursts (Weilbacher et al., 2000).

\subsection{Optical Spectroscopy}

The main motivation for our spectroscopic campaign was to confirm the association of the selected TDG candidates with the main interacting galaxy and to determine their metallicities and internal extinction values to further refine our models, and to exclude low metallicity dwarf galaxies in the neighbourhood of the interacting galaxies. We therefore chose to observe the whole optical wavelength range with low resolution, including all important emission lines from [OII] 3727 to [SII]6717,6731 (Weilbacher et al., 2003).

While we could not get redshifts for all TDG candidates due to constraints of the slit placements in our multi-object spectroscopy, and in very few cases also because of low $\mathrm{S} / \mathrm{N}$ of the resulting spectra, we could confirm the association of 29 TDG candidates with their parent galaxies. This proves our method with color selection by evolutionary models as a very successful pre-selection for TDG candidates. All of the TDG candidates for which we actually obtained a spectroscopic redshift are related to the central galaxy!

The metallicity measurement was carried out with various methods used in the literature for optical emission line spectra, ranging from the $P / P_{3}$ method of Pilyugin (2001), to the "standard" $R_{23}$ method, and the [NII] method of van Zee et al.(1998). Only in one case could we use the physical method (Shields, 1990), because the weak line [OIII]4363 was undetected in all other cases. We compared the results of these methods, and if they agreed within their error bars we selected the value given by the method with the smallest systematical error. This way, we found three galaxies with $12+\log (O / H)<8.0$ which we excluded 
as TDG candidates because "recycled" gas cannot allow such low metallicity TDGs to form. We are therefore left with 26 TDG candidates. These have a mean oxygen abundance in the range $12+\log (O / H)=8.34 \pm 0.14$ or about $1 / 4 Z_{\odot}$, absolute magnitudes of $M_{B}=-12 \ldots-17 \mathrm{mag}$, and $\mathrm{H} \alpha$ luminosities between those of the most luminous Hir regions of spiral galaxies and those of "normal" dwarf galaxies.

Finally, and a bit to our surprise, we could also derive velocity curves of the ionized gas within some of our TDG candidates even at the low spectral resolution used $(R<400)$. Velocity differences $\Delta V_{\max }$ we measure within these TDGs can reach up to $\sim 500 \mathrm{~km} \mathrm{~s}^{-1}$. While we do not think that it would be correct to convert the measured values of $\Delta V_{\max }$ into dynamical mass estimates, and the origin of these apparent velocity gradients is not clear, it seems reasonable to assume that these knots are kinematically decoupled from the tidal tail, i.e. real TDGs.

One of the interacting galaxies from our sample, AM 1353-272 which we dubbed "The Dentist's Chair" due to its appearance on optical images, was reobserved with FORS2 on the ESO VLT at higher spectral resolution $(R \approx$ $700)$. Curved slits allowed us to cover both tidal tails of this system and we indeed observed significant velocity gradients in 7 TDGs in this one interacting system (Weilbacher et al., 2002) and $\Delta V_{\max }$ in the range 24 to $343 \mathrm{~km} \mathrm{~s}^{-1}$ ! This gives us a total number of 13 TDGs with hints of kinematical independence, for now a lower limit which has to be checked with more spectroscopic data and higher resolution.

\subsection{Near-Infrared Photometry \& Refined Models}

Our near-infrared (NIR) photometry is mainly derived from deep $H$-band images taken with the SOFI camera on the ESO NTT. Two of our interacting systems were also observed in $J$, and three in the $K s$-band. NIR-observations are particularly important to constrain the contribution of old stars, inherited from the parent galaxy, as these are hidden in the current starburst at optical wavelengths while they possibly dominate the NIR luminosities.

This data together with our previous observations gives us the spectral energy distribution of all our TDGs and TDG candidates in the wavelength range from $\sim 0.43$ to $2.16 \mu \mathrm{m}$. From spectroscopy we have another observable, the $\mathrm{H} \beta$ equivalent width, so that we can altogether compare up to 7 observed properties $\left(B, V, R, J, H, K_{\mathrm{s}}, \mathrm{H} \beta\right)$ with our grid of evolutionary synthesis models.

To compare the data to the models we developed a least-squares based procedure, to identify the model that best represents the observed data in an objectively reproducible way. First, we deredden the observed colors using the spectroscopic estimate of the total extinction. Next, we select the model(s) with the metallicity matching the observed oxygen abundance. Then, we select the timesteps of each model where $\operatorname{EW}(\mathrm{H} \beta)$ matches the observed value within the error bars, and evaluate how close the model is to the observed SED for each timestep of these selected models. Finally, we derive the interesting properties from the model and timestep best matching the observed properties.

This procedure confirms the rough estimates we previously had from the comparison with the optical data: our TDGs show young burst ages, mostly up to $20 \mathrm{Myr}$, some even seem to be at the beginning of their starburst, i.e. before 


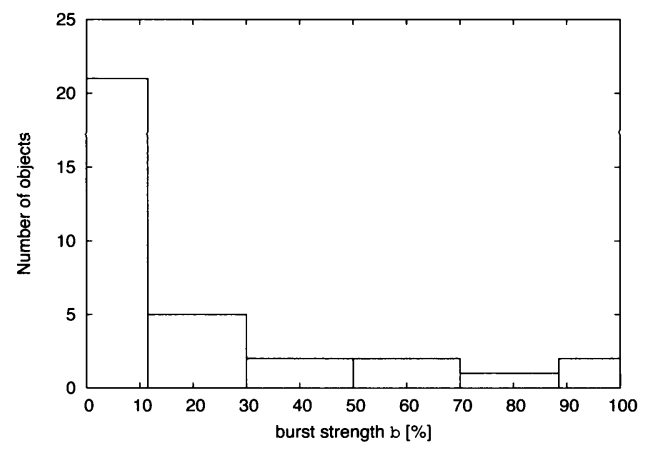

Figure 1. A histogram of the current burst strength derived from the comparison of evolutionary models with the data of our sample of TDGs.

reaching the maximum star formation rate. The model also allows us to derive a good estimate of the total stellar masses which have a mean value of $\sim 2 \times$ $10^{8} M_{\odot}$, typical for dwarf galaxies. We also estimate the current star formation rate (SFR) in the TDGs, circumventing the problems with the usual $L(\mathrm{H} \alpha)$ conversion (Weilbacher \& Fritze-von Alvensleben, 2001). The mean SFR of $0.05 M_{\odot} \mathrm{yr}^{-1}$ shows them to be strongly star-forming galaxies in relation to their sizes and masses.

Most importantly, we also derive the burst strengths $b$ : Fig. 1 show the distrubtion of burst strenghts of the TDG candidates in our sample. Their current burst strength $b$ indeed covers the entire range of possible values from 0 to $100 \%$, with a strong peak in the lowest bin $(b<10 \%)$. This shows that many TDGs in our sample have most likely formed from stellar condensations and only few of them from the collapse of giant gas clouds (Weilbacher et al., in prep.).

\section{Conclusions}

We used a three stage approach of optical photometry, optical spectroscopy, and near-infrared photometry combined with dedicated evolutionary synthesis models to select a sample of TDG (candidates), and study their gaseous properties and stellar populations.

We selected 44 TDG candidates from optical imaging in comparison with our models from over 100 knots in the tidal tails of our sample of 14 interacting and merging galaxies. From optical spectroscopy we confirm the association of the TDG candidates with the parent galaxies. The metallicity measurement shows them to have a mean oxygen abundance close to $1 / 4 Z_{\odot}$, while three TDG candidates were rejected due to their too low metallicities. Low resolution spectroscopy also revealed 13 TDGs to show kinematical signatures independent of their surrounding tidal tail. We therefore roughly find at least one true TDG per interacting system. 
From our near-infrared analysis and the detailed comparison of their spectral energy distributions with our evolutionary synthesis models we confirm that the TDGs in our sample indeed have the stellar masses of dwarf galaxies and show strong star-formation activity. Their burst strengths show most of them to have primarily formed from stellar condensations and only a smaller percentage from the collapse of giant gas clouds.

Acknowledgments. PMW's work on this project was partially supported by the DFG (grants FR 916/6-1 and 916/6-2). Further support by the European Research and Training Network Spectroscopic and Imaging Surveys for Cosmology under contract HPRN-CT-2002-00316 is acknowledged. UFvA gratefully acknowledges partial travel support from the IAU \& DFG (Fr 916/12-1).

\section{References}

Arp, H. \& Madore, B. 1987, A Catalogue of Southern Peculiar Galaxies and Associations (Cambridge: Cambridge University Press)

Barnes, J. \& Hernquist, L. 1992, Nature, 360, 715

Conselice, C. J., Bershady, M. A., Dickinson, M., \& Papovich, C. 2003, AJ, 126, 1183

Duc, P.-A., Brinks, E., Springel, V., Pichardo, B., Weilbacher, P., Mirabel, I. F. 2000, AJ, 120, 1238

Duc, P.-A. \& Mirabel, I.F. 1994, A\&A, 289, 83

Duc, P.-A. \& Mirabel, I.F. 1998, A\&A, 333, 813

Elmegreen, B., Kaufman, M., \& Thomasson, M. 1993, ApJ, 412, 90

Hibbard, J., Guhathakurta, P., van Gorkom, J., \& Schweizer, F. 1994, AJ, 107, 67

Okazaki, T. \& Taniguchi, Y. 2000, ApJ, 543, 149

Pilyugin, L. 2001, A\&A, 369, 594

Shields, G. 1990, ARA\&A, 28, 525

van Zee, L., Salzer, J., Haynes, M., O’Donoghue, A., \& Balonek, T. 1998, AJ, 116,2805

Weilbacher, P. M. 2002, PhD Thesis, Universität Göttingen

Weilbacher, P. M. \& Duc, P.-A. 2001, in Dwarf Galaxies and their Environment, ed. K. de Boer, R.-J. Dettmar, \& U. Klein, 269-272

Weilbacher, P. M., Duc, P.-A., \& Fritze-von Alvensleben, U. 2003, A\&A, 397, 545

Weilbacher, P. M., Duc, P.-A., Fritze-von Alvensleben, U., Martin, P., \& Fricke, K. J. 2000, A\&A, 358, 819

Weilbacher, P. M. \& Fritze-von Alvensleben, U. 2001, A\&A, 373, L9

Weilbacher, P. M., Fritze-von Alvensleben, U., Duc, P.-A., \& Fricke, K. J. 2002, ApJ, 579, L79

Zwicky, F. 1956, Erg. d. exakt. Naturwiss., 29, 344 